\title{
Clinical Studies Evaluating Effects of Probiotics on Parameters of Intestinal Barrier Function
}

\author{
Saskia van Hemert ${ }^{1 *}$, Jurre Verwer ${ }^{1}$, Burkhard Schütz ${ }^{2}$ \\ ${ }^{1}$ Winclove Probiotics, Amsterdam, the Netherlands \\ ${ }^{2}$ Biovis Diagnostik MVZ GmbH, Limburg-Offheim, Germany \\ Email: *Saskiavanhemert@winclove.nl
}

Received March 6, 2013; revised April 4, 2013; accepted May 4, 2013

Copyright (C) 2013 Saskia van Hemert et al. This is an open access article distributed under the Creative Commons Attribution License, which permits unrestricted use, distribution, and reproduction in any medium, provided the original work is properly cited.

\begin{abstract}
The intestinal barrier is important in preventing translocation of bacteria, toxins and antigens from the lumen of the gut into the body. Enhanced permeability, or gut leakiness, has been associated with different diseases. Probiotics can, strain-specifically, improve the epithelial barrier function. However, so far most researches have used cell lines or animal models due to the difficulty of measuring the effects of products on the epithelial barrier function in vivo in humans. Here a systematic literature search was performed to find articles addressing the effects of probiotics on the barrier function in human trials. The Pubmed database was searched (January 2013) to identify human in vivo studies with probiotic products in which parameters for epithelial barrier function were measured. In total 29 studies were identified, but patients, bacterial characteristics and methods to measure intestinal barrier function caused large heterogeneity among these studies. About half of the studies showed positive results of probiotics on the epithelial barrier function, indicating a clear potential of probiotics in this field. In a case series of 14 patients using Ecologic ${ }^{\circledR} 825$, a probiotic food supplement with known effect on epithelial barrier function, different markers of intestinal integrity improved significantly. Further studies in this field should consider strain(s), dose and duration of the probiotic supplementation as well as the markers used to measure epithelial barrier function. Besides the lactulose/mannitol test, zonulin and $\alpha 1$-antitrypsin might be valuable markers to measure epithelial barrier function in future experiments.
\end{abstract}

Keywords: Bacteria; Epithelial Barrier; Gut Permeability; Intestinal Barrier Function; Intestinal Integrity; Probiotics; Review; Vivo Studies

\section{Introduction}

The intestinal epithelial cells have a dual function. On one hand they facilitate absorbance and transport of nutrients, electrolytes and water. On the other hand they also form a barrier between the body and the bacteria, toxins and antigens present in the gut. The ability to control the invasion of harmful content from the lumen to the body is called the intestinal barrier function. This defense mechanism consists of multiple elements, like the mucous layer, secretory IgA, antimicrobial peptides, and the apical junction complex. The latter is composed of tight junctions, adherence junctions and desmosomes. The tight junctions seal the paracellular pathway and are the rate-limiting step in the transport between adjacent epithelial cells [1].

The intestinal barrier can be disturbed by various causes, like certain medicines, exercise, mast cell activation, high

"Corresponding author. fat diet, stress, etc. [1]. This can lead to an increased permeability, allowing amongst others enhanced entrance of lipopolysaccharides (LPS) into the body. LPS are parts of the outer membrane of Gram-negative bacteria and are strong endotoxic compounds. They can cause the release of pro-inflammatory cytokines in the body, leading to inflammation. Increased permeability of the epithelial barrier has been associated with many gastrointestinal inflammatory disorders, like inflammatory bowel diseases (IBD, Crohn's disease and ulcerative colitis), irritable bowel syndrome (IBS), food allergies and celiac disease [2]. In addition, increased permeability can also lead to systemic inflammatory diseases, like allergy, metabolic syndrome, diabetes, atherosclerosis, chronic fatigue syndrome, autism, migraine and rheumatoid arthritis [3].

Probiotics are living microorganisms that have beneficial effects on the health of the host [4]. Strains documented as probiotic tend to be species of Lactobacillus or Bifidobacterium. More and more is known that probiotic 
effects are species- and even strain-specific. Certain probiotics have shown to be effective in different gut-related diseases, like antibiotic-associated diarrhea and necrotizing enterocolitis in premature infants $[5,6]$. For some other diseases, including IBD the effects of probiotics are promising, but so far results from clinical trials have been inconsistent [7]. Probiotics have proven strain-dependent capabilities in vitro as well as in vivo to improve the epithelial barrier function via different mechanisms $[8,9]$. Most work has been done in cell culture systems or in animal models [9-11]. Effects in humans are difficult to measure in vivo due to the inaccessibility of the intestine. One way to overcome this problem is by oral administration of test substances and measurement of urinary excretion. The lactulose/mannitol $(\mathrm{L} / \mathrm{M})$ test or a comparable sugar test is the most used method to measure intestinal permeability. Lactulose is a disaccharide which is passively absorbed through the paracellular pathway via the tight junctions. Mannitol, which is a monosaccharide, is transported via the transcellular pathway. In the case of heightened permeability, more lactulose passes the barrier and eventually ends up in the urine. Therefore, a high lactulose/mannitol ratio represents a high, i.e. pathological, intestinal permeability. Other test substances that are used, are ${ }^{51} \mathrm{Cr}$-EDTA or polyetylene glycols (PEG). The ${ }^{51} \mathrm{Cr}$-EDTA test has been used to detect increased intestinal permeability in Crohn's Disease, celiac disease, and non-alcoholic fatty liver disease [12-14]. Urinary excretion of PEG was significantly higher in patients with alcoholic liver disease and with acute pancreatitis compared to healthy controls $[15,16]$. Disadvantages are that all these methods are not very sensitive, and that they are affected by intestinal transit and the renal elimination rate [16]. Alternatively, other biomarkers for intestinal permeability are sometimes measured, like intestinal fatty acid binding protein (IFABP), C-reactive protein (CRP), tumor necrosis factor $\alpha$ (TNF$\alpha$ ), alpha1-antitrypsin, calprotectin, eosinophil cationic protein (ECP) and zonulin [17,18]. These biomarkers have been correlated with barrier function, but do not measure it directly. IFABP is a protein specifically located in the apical villi of small bowel mucosa that is released into the systemic circulation in the event of enterocyte death and is a marker for enterocyte damage and intestinal ischemia $[19,20]$. CRP, TNF- $\alpha$ and ECP are general markers for inflammation, whereas $\alpha 1$-antitrypsin and calprotectin are markers for intestinal inflammation. Zonulin is a relatively novel marker of permeability [3]. It is a physiologic modulator of the intercellular regulation of tight junction proteins and thereby the paracellular epithelial intestinal permeability [21-23]. Moreover, the intestinal permeability can also be assessed by measuring the bacterial translocation (BT) through analysis of mesenteric lymph nodes (MLN) or blood plasma by quantitative or qualitative PCR.
In this review an overview of human studies is given in which effects of probiotics on the intestinal barrier function are investigated. Also a case series with Ecologic ${ }^{\circledR} 825$ is described, a potential probiotic product which has shown positive effects on epithelial barrier function in both a cell culture system (Saskia van Hemert, personal communication), as well as in a rat model of chronic water avoidance stress [24].

\section{Material and Methods}

\subsection{Literature Review}

The systematic literature search was conducted in PubMed up to 1 January 2013, using the following (truncated) keywords "probiotic*, lactobacill* or bifidobact* AND trial AND barrier or permeability". A second search was performed with "probiotic*, lactobacill* or bifidobact* AND epithelial barrier, intestinal integrity or tight junctions". The search was limited to full-text English written papers. This resulted in 205 hits. By screening the titles and abstracts, studies performed in vitro, in animals and reviews were subsequently excluded. Full text of the remaining 25 papers was checked. Additional papers were found by checking references of pertinent articles and by searching on "probiotic*" and different methods of measuring intestinal permeability.

\subsection{Case Series}

In the case series 14 patients, 3 male and 11 female, between 18 and 65 (mean 46) years of age were included in September 2011. The participants were informed by their physician and gave their informed consent to the physician. All procedures were in accordance with the Helsinki Declaration of 1975. Exclusion criteria were known inflammatory gastrointestinal diseases, use of probiotics or antibiotics four weeks prior to the study, use of gastric acid inhibitors, presence of known diabetes type II and pregnancy. The patients were supplemented with a daily dose of $>7.5 \times 10^{9}$ cfu Ecologic ${ }^{\circledR} 825$ (Winclove Probiotics) for 8 weeks. Ecologic ${ }^{\circledR} 825$ is a food supplement containing a mixture of 9 bacterial strains: Bifidobacterium bifidum W23, Bifidobacterium lactis W51, Bifidobacterium lactis W52, Lactobacillus acidophilus W22, Lactobacillus casei W56, Lactobacillus paracasei W20, Lactobacillus plantarum W62, Lactobacillus salivarius W24 and Lactococcus lactis W19. Before the start of the supplementation ( $t=0$ weeks) and at the end $(t=8$ weeks) blood serum and faeces samples were collected to determine parameters for impaired barrier function.

\subsection{Analyses}

Zonulin, $\alpha 1$-antitrypsin and calprotectin in stool samples were analyzed with commercially available ELISA kits (Immundiagnostik AG, Bensheim, Germany) as described 
earlier [25]. In the serum levels the following parameters were measured with standard procedures (Biovis Diagnostik): IgG4 antibodies against banana, egg, hazelnut, cow milk, soy beans and wheat, and high sensitive (hs)CRP. IgG4 antibodies were measured by DST Ag Schwerin and the hsCRP on Siemens Immulite 2000. A lactulose/mannitol test was performed by giving the patients a mixture of $30 \mathrm{mg}$ mannitol and $150 \mathrm{mg}$ lactulose stirred into $50 \mathrm{~mL}$ non-carbonated mineral water. Urine samples were analysed by applying the HPLC method of Immuchrom GmbH Heppenheim.

\subsection{Statistics}

For the case series, a paired student t-test was used to measure the significance of the parameters before and after the intervention period. Differences were considered significant at 2-tailed $\mathrm{p}<0.05$.

\section{Results and Discussion}

\subsection{Literature Study}

In total 29 studies were found by systemic literature search, which investigated the effect of a single bacterial strain or a bacterial mixture on the human intestinal barrier function in vivo (Tables $\mathbf{1}$ and 2). This was often a secondary endpoint, as the primary endpoint in most cases was a decrease in a clinical endpoint. More than half of the studies (17/31) showed a positive effect of the used probiotics on epithelial barrier function, whereas the other studies (13/31) did not show an effect. One study had two arms and a placebo whereby a positive effect was found in only one of the arms [26]. In general, the study groups were small, ranging from 4 to 81 persons per group. For some studies the small numbers of participants might be an explanation for the fact that no significant effects were found, for example the study of Alberda et al., showed a trend ( $\mathrm{p}=0.06)$ with groups of 7 patients only [28]. Most studies $(n=20)$ had treatment or alleviation of clinical symptoms as purpose, while a small group of studies $(n=9)$ aimed for a preventive effect $[18,28-35]$. This effect was sought for example in preventing bacterial infections in infants, [33] or preventing post-infectious complications [18,30-32,34]. At first sight, the result consists of a very heterogeneous assembly of investigations. This variety is reflected in a number of factors related to the probiotics, to the patient population and the methodology. The probiotics used in the studies varied in daily dose, duration of supplementation and number and type of bacterial strains. The patient population ranged from healthy volunteers to critically ill patients and preterm infants. The use of medicines like non-steroid anti-inflammatory drugs and antibiotics are an additional factor of variation in the patient population. There were also marked differences between the power of the studies, methodological quality of the studies and the analytical methods to measure intestinal barrier function. The heterogeneity found in this literature review, is also found in other recent reviews summarizing for example the role of probiotics in the treatment of eczema, IBD and IBS [36-38]. The heterogeneity caused by the probiotic regime, the patient population and the methodology of measuring epithelial barrier function will be discussed below.

\subsubsection{Probiotic Regime}

One of the variables related to the used probiotics is the daily dose. This ranged from $1 \times 10^{9}$ colony forming units (CFU) [28] to $1 \times 10^{12} \mathrm{CFU}$ [35] of the studies yielding positive effects and from $1 \times 10^{9} \mathrm{CFU}$ [39] to 9 $\times 10^{11}$ CFU [27] of the studies with no clear benefit on barrier function. When looking at the mean daily dose, the positive studies administered on average $1 \times 10^{11}$ CFU, while the studies without positive effects used $8 \times$ $10^{10} \mathrm{CFU}$, a negligible difference on the log-scale used to determine the CFU. Also the medians were very similar, $1 \times 10^{10} \mathrm{CFU}$ in both groups. These results imply that a relatively high dose is not principally necessary to result in a positive effect nor does it give any guarantee of such. Moreover, different patient groups might benefit from different daily doses to elicit effects.

The duration of supplementation in the studies also varied strongly, from 1 [35] to 183 days [40] in the positive studies and from 2 [41] to 92 day [42] in the studies without an effect. The mean durations were 40 vs. 28 days for studies with a positive effect and no effect respectively, whereas the medians were 28 days for both groups of studies. Some authors suggested that the lack of a positive outcome was due to a too short intervention duration [31,43]. However other longer studies did not find any positive effect as well [42,44]. One interesting finding is the loss of the beneficial effect over time. In the study of Gupta et al. [40] a positive effect on intestinal permeability was found after 12 weeks, but this effect was lost after 24 weeks, despite a supplementation during 24 weeks. Finally, studies were also checked for a follow-up period, to speculate on the persistence of a beneficial effect of probiotics. Only two studies specifically defined a follow-up test but neither of both tested the intestinal permeability at that time $[45,46]$. Although the clinical symptoms remained lower in the probiotics group, nothing can be said about the persistent effects on intestinal permeability.

From in vitro studies it is already known that bacterial strains can vary considerably on their effects on epithelial barrier function [8,47]. Also the studies in this review used a variety of strains whereby L. rhamnosus (mainly LGG) and L. plantarum were the most used species. Strain selection is an important point in the design of studies with probiotics as the effects can vary largely 


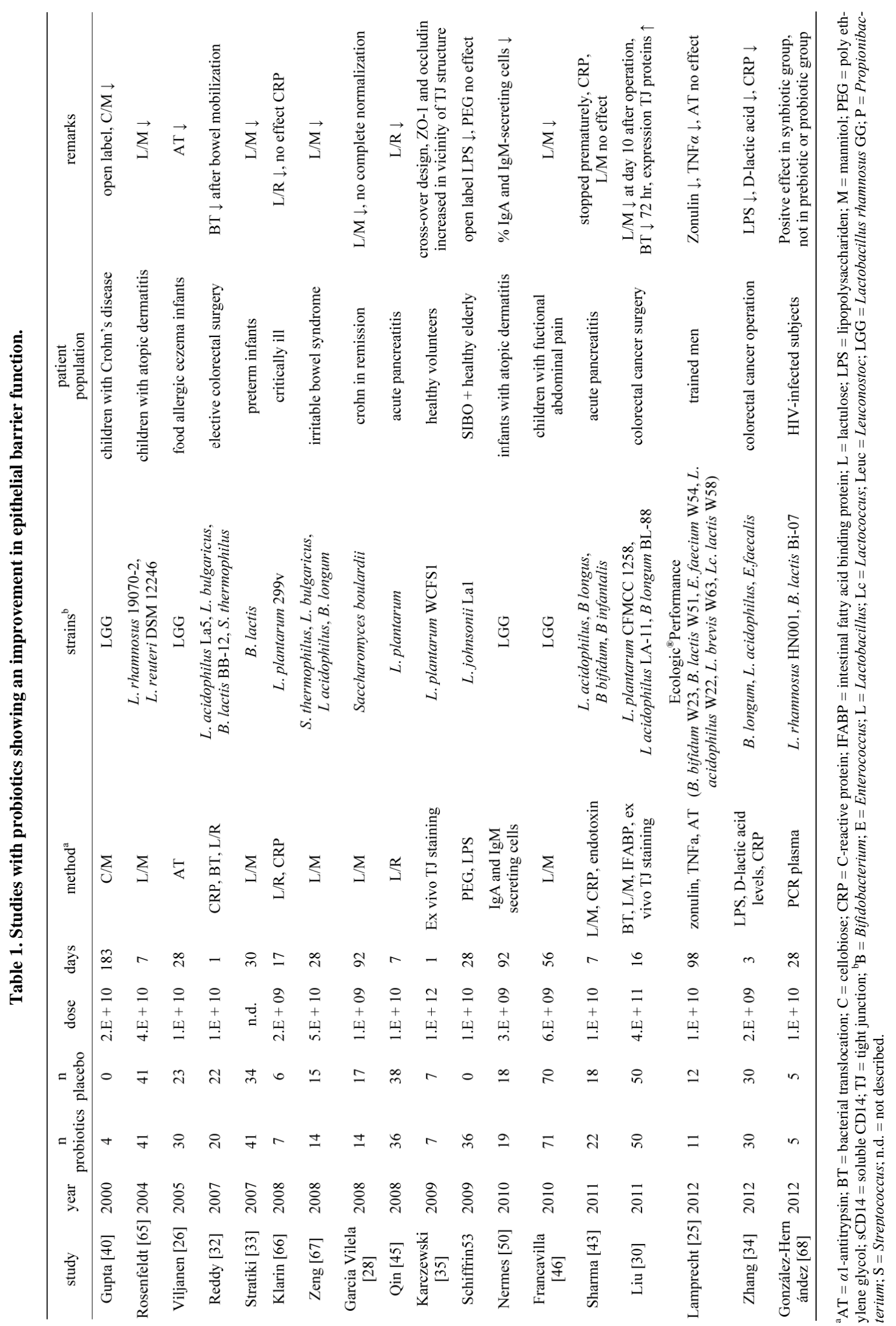




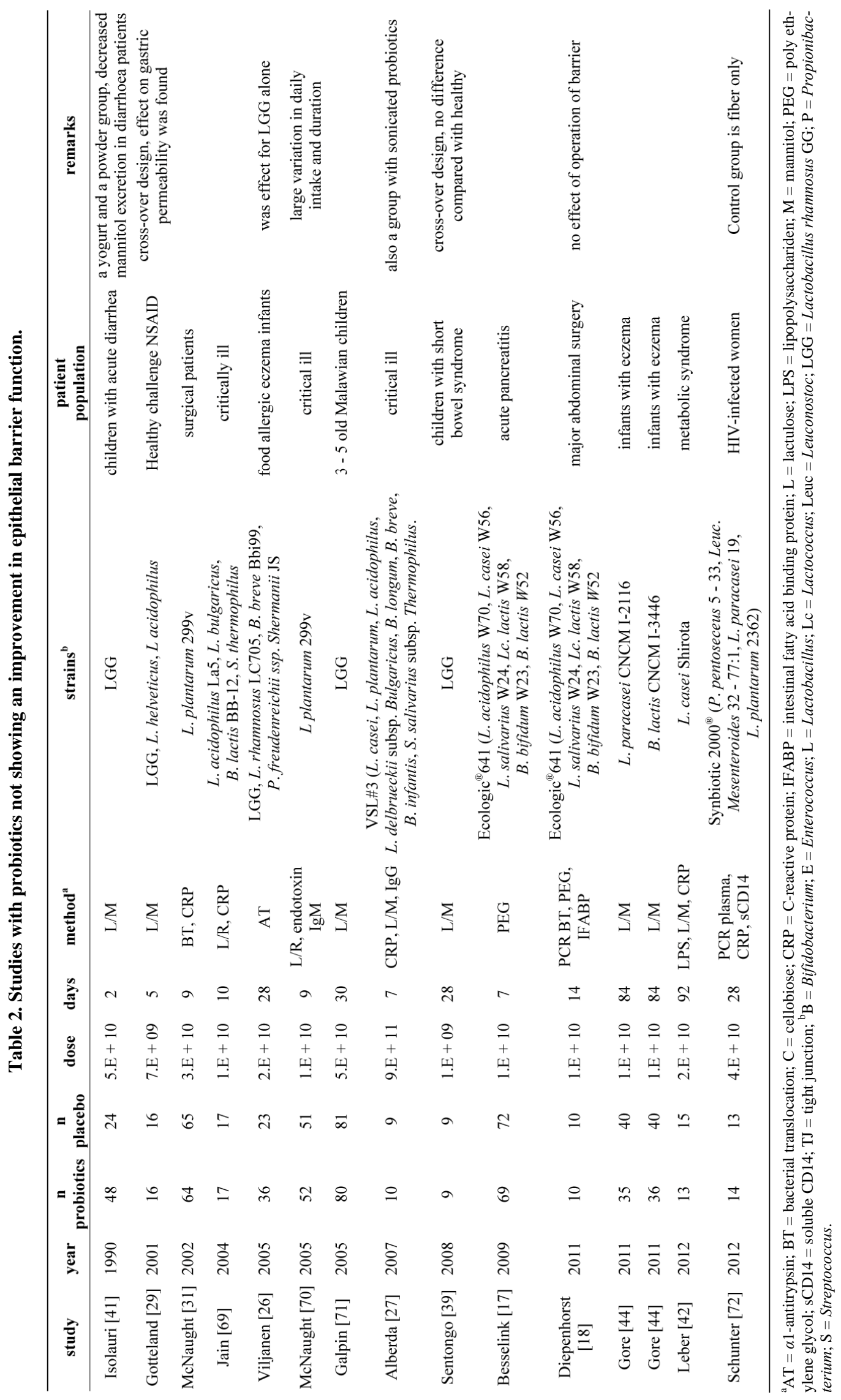


between strains. It has been suggested that multispecies probiotic products (products with multiple strains, preferentially of different genera) are in general more effective compared to monospecies products (containing a single strain) $[48,49]$, but for the studies in this review the results were comparable for monostrain and multispecies products. Monostrain products were used in 10 studies with a positive outcome and 8 studies without a beneficial effect on barrier function, whereas multispecies products were involved in 8 studies with a positive outcome and 7 studies without beneficial effect. However, for the large majority of the studies it was not described how the used strains were selected or why these specific strains were used.

\subsubsection{Patient Groups}

Another point of interest is the variation of patient groups in the studies reviewed. While patients with atopic eczema/dermatitis suffer from inflammatory processes leading to or even might be caused by barrier dysfunctions in the skin and gut, the clinical manifestation in acute pancreatitis or other critically ill patients is quite different. Different pathological mechanisms can lead to various problems with the epithelial barrier, perhaps requesting diverse probiotic approaches.

\subsubsection{Measurement of Intestinal Permeability}

Besides heterogeneity in the bacterial strains and the patient group, the method of measuring the intestinal permeability was a third source of heterogeneity. While the majority of the studies used a sugar absorption test (19 out of 29), the used sugars were variable (lactulose, mannitol, cellobiolose, sucrose or rhamnose). Other methods were PEG and in some studies tight junction protein staining was used. IFABP or IgA/IgG were additionally measured in some studies $[17,18,50]$ as indirect indices of the epithelial barrier integrity. CRP, TNF- $\alpha, \alpha 1$-antitrypsin, mesenteric lymph nodes biopsies and PCR were often used to assess inflammatory and/or bacterial activity or translocation. Measuring bacterial translocation to the mesenteric lymph nodes can only be done in studies with operations. This method does not seem to correlate with results of a sugar test [51] and it has been suggested that bacterial translocation after abdominal surgery may be part or normal antigen-sampling processes of the gut [18]. The two studies with HIV-infected individuals both used PCR on blood plasma to determine microbial translocation, whereas this method has not been used in any of the other studies with probiotics. Whereas this method is relevant to measure intestinal barrier function remains to be determined, as liver function might also influence the levels of bacterial DNA in blood plasma. A relatively novel marker of barrier function is zonulin, which was used in a recent study with probiotics [25]. Zonulin has been suggested as one of the most valid surrogate mark- ers to estimate intestinal barrier function in humans [52].

There is no evidence that one specific method is more sensitive than another. In studies using the $\mathrm{L} / \mathrm{M}$ ratio an effect was not found more often than studies using other sugar tests. However, in all studies using PEG no effect was observed $[17,18,53]$. Interestingly in one study effects of probiotic supplementation on plasma levels of LPS were observed, despite a lacking effect as measured with PEG [53]. Although the L/M test or a comparable sugar test was often used, this method had some drawbacks. First, due to the lack of a standardization [54], the $\mathrm{L} / \mathrm{M}$ ratio varies considerably even among healthy individuals. Indeed, the investigators of the current articles differ in their normal values of the $\mathrm{L} / \mathrm{M}$ ratio regarding what is physiological and pathological. Second, the quantity of sugars used varied from $250 \mathrm{mg}$ to $10 \mathrm{~g}$ for lactulose and from $100 \mathrm{mg}$ to $5 \mathrm{~g}$ for mannitol. Although the absolute quantities used might not be of major influence, as the eventual ratio is a relative value, the osmolarity of the test solution can have influenced the outcomes [54]. Other methods to measure intestinal permeability are ${ }^{51} \mathrm{Cr}$-EDTA and ${ }^{99} \mathrm{mTCDTPA}$, which are nondegraded radio-labeled chelates used to measure intestinal permeability [55], but so far they have not been used by studies with probiotics.

\subsection{Case Series}

To investigate which parameters are useful to measure in relation to probiotics and epithelial barrier function, we measured different parameters related to barrier function in a case series of 14 patients.

Zonulin levels in faeces were elevated $(>30 \mathrm{ng} / \mathrm{ml})$ in 10 of the 14 people participating in the case series before the start of the intervention. The average zonulin levels decreased significantly after the 8 weeks intervention period ( $p<0.01$, Figure 1(a)). In the group of people started with elevated zonulin levels, the levels decreased in 8 of the 10 persons during the intervention period. In the control group 2 of the 4 persons showed decreased zonulin levels after 8 weeks of intervention. Alpha1antitrypsin levels were elevated in 7 out of the 14 people before the start of the intervention period $(>27.2 \mathrm{mg} / \mathrm{dl})$ and it decreased significantly after the intervention $(\mathrm{p}<$ 0.01 , Figure 1(b)). These levels were decreased in 6 of the 7 people starting with elevated levels, and also in 6 of the 7 people starting with levels in the normal range $(<27.2 \mathrm{mg} / \mathrm{dl})$. Interestingly the person who started with elevated levels of alpha1-antitrypsin, but didn't show a decrease also did not show a decrease in zonulin. The three persons who had the highest zonulin levels before the start of the study also had the highest alpha1-antitrypsin at the start. High sensitive CRP was measured in only 9 people, due to technical problems. The levels were elevated in 5 of the 9 people before the start of the trial 

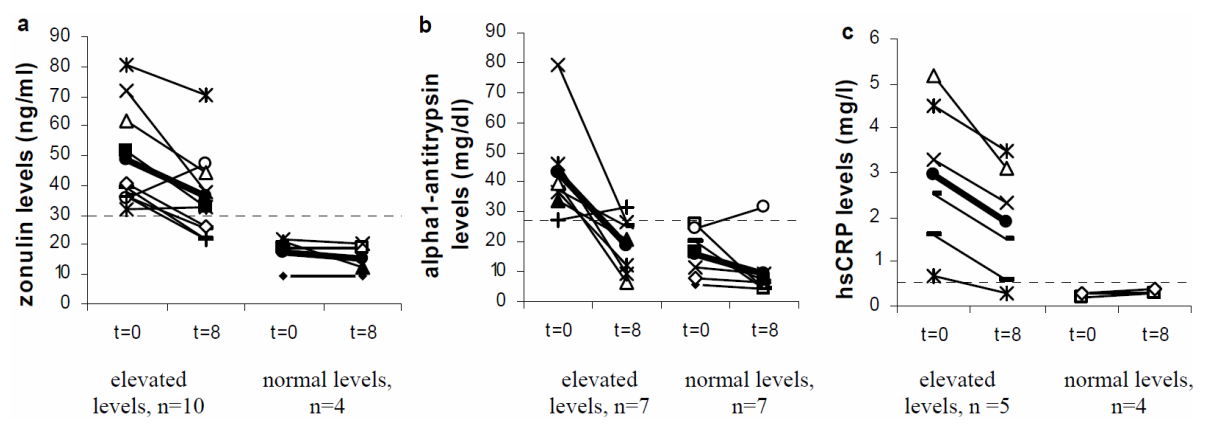

Figure 1. Effect of 8 weeks supplementation with Ecologic ${ }^{\circledR} 825$ on the average levels of (a) zonulin; (b) $\alpha 1$-antitrypsin and (c) hsCRP. Normal values are indicated with a dotted line. The average values are indicated in bold •.

$(>0.55 \mathrm{mg} / \mathrm{l})$. Those levels were decreased in all five participants which had elevated levels before the start of the intervention, but not in the persons which started with normal values (Figure 1(c)). The IgG4 antibodies against food allergens (measured in 9 of the 14 people) did not show any differences before and after the intervention (results not shown). Calprotectin levels were in all cases under the detection limit of $10 \mathrm{mg} / \mathrm{l}$. In addition there were also no differences found with the L/M test.

The participants in these case series visited their physician due to gastrointestinal complaints. In our study elevated levels of three markers of intestinal permeability were found in the majority of the participants. These markers were zonulin, $\alpha 1$-antitrypsin and CRP. In this cases series, zonulin levels were measured in faeces, as was also done in a study investigating the effects of probiotic supplementation in trained men [24]. Measurements of zonulin have also been performed is intestinal tissue and serum. Tissue levels of zonulin in the intestine were much higher in celiac patients compared to controls [21]. Higher levels of zonulin in serum correlate with higher intestinal permeability as measured with the $\mathrm{L} / \mathrm{M}$ test $[56,57]$. This is in contrast with our study, where no abnormal L/M ratio was measured while we did find changes in zonulin levels. This might indicate that zonulin is a more sensitive marker of intestinal barrier function than the $\mathrm{L} / \mathrm{M}$ test.

Alpha1-antitrypsin is a marker used as a measure of protein leakage into the intestinal tract and for inflammation in individuals with IBD [58]. In the case series a significant decrease in the levels of $\alpha 1$-antitrypsin in faeces was detected. A similar decrease of levels of $\alpha 1$-antitrypsin was found in a study with probiotics in children with cow-milk allergy [26], but not in a study with probiotics in trained men [25]. This latter finding might be due to the fact that the $\alpha 1$-antitrypsin levels were not elevated before the start of the study in the study population, whereas this was the case in the described case series and the study of Viljanen et al. [26]. As inflammation is linked to impaired intestinal barrier function [59] it is not surprising that a marker for inflammation might also be valuable as a marker for barrier function. Faecal calprotectin is, similar as $\alpha 1$-antitrypsin, used as marker in IBD [60], but the levels of calprotectin were below the detection limit of $10 \mathrm{mg} / \mathrm{L}$ in the studied patient group. This might indicate that in contrast to $\alpha 1$-antitrypsin, calprotectin is not a useful marker for subtle intestinal barrier problems, but more relevant in diseases like IBD with marked inflammation of the epithelial barrier function. Another marker for inflammation is CRP, which was also identified in the case series. In another study CRP tended to be higher in obese patients compared with healthy weight controls [61]. CRP levels were associated with certain faecal metabolites and were inversely correlated with the total microbial counts, indicating a possible influence of the gut microbiota on CRP levels. Elevated CRP levels were correlated with enhanced L/M ratios in some studies [62,63], but not in all [64]. This suggests that increased levels might be caused by enhanced intestinal permeability, but can also have other causes. These elevated levels of CRP might indicate a systemic response to increased LPS in the body due to an enhanced permeability of the gut. From that perspective, CRP is not a direct measurement of intestinal permeability, but is probably more an indirect measurement.

\subsection{Wider Implications}

The primary aim of this narrative review was to provide an intensive overview of the current literature regarding the influence of probiotics on the human intestinal barrier function. This review is, to our knowledge, the first to overview the literature of probiotics and intestinal permeability in in vivo human studies. Overall, there are several indications in vivo that probiotics can have positive effects on the barrier function, as a positive effect was found in $48 \%$ of the controlled studies. The heterogeneity of the studies makes it impossible to draw any conclusions on probiotic treatment specifications. It is plausible that different patient groups might benefit from different treatment plans, such as daily dose, strain and duration. Moreover, different disturbances of the gut barrier might also ask for different barrier measurements. The case series indicated that zonulin, $\alpha 1$-antitrypsin and 
hsCRP might be valuable markers to measure intestinal permeability in vivo. Clearly, more investigations have to be conducted to draw strong conclusions. For these investigations consensus is necessary on standardized methods to measure barrier function.

\section{Acknowledgements}

The authors like to thank the participants of the case series for their participation in the study.

\section{REFERENCES}

[1] A. Farhadi, A. Banan, J. Fields and A. Keshavarzian, "Intestinal Barrier: An Interface between Health and Disease," Journal of Gastroenterology and Hepatology, Vol. 18, No. 5, 2003, pp. 479-497. doi:10.1046/j.1440-1746.2003.03032.x

[2] A. M. Marchiando, W. V. Graham and J. R. Turner, "Epithelial Barriers in Homeostasis and Disease," Annual Review of Pathology-Mechanisms of Disease, Vol. 5, 2010, pp. 119-144. doi:10.1146/annurev.pathol.4.110807.092135

[3] A. Fasano, "Leaky Gut and Autoimmune Diseases," Clinical Review of Allergy and Immunology, Vol. 42, No. 1, 2012, pp. 71-78. doi:10.1007/s12016-011-8291-X

[4] FAO/WHO, "Evaluation of Health and Nutritional Properties of Probiotics in Food Including Powder Milk with Live Lactic Acid Bacteria,” 2001.

[5] E. J. Videlock and F. Cremonini, "Meta-Analysis: Probiotics in Antibiotic-Associated Diarrhoea," Alimentary and Pharmacology Therapy, Vol. 35, No. 12, 2012, pp. 13551369. doi:10.1111/j.1365-2036.2012.05104.x

[6] K. Alfaleh, J. Anabrees, D. Bassler and T. Al-Kharfi, "Probiotics for Prevention of Necrotizing Enterocolitis in Preterm Infants," Cochrane Database Systematic Reviews, No. 3, 2011, Article ID: CD005496. doi:10.1002/14651858.CD005496.pub3

[7] I. R. Rowland, et al., "Current Level of Consensus on Probiotic Science-Report of an Expert Meeting-London, 23 November 2009," Gut Microbes, Vol. 1, No. 6, 2010, pp. 436-439. doi:10.4161/gmic.1.6.13610

[8] E. Miyauchi, et al., "Mechanism of Protection of Transepithelial Barrier Function by Lactobacillus Sali- varius: Strain-Dependence and Attenuation by Bacteriocin Production," American Journal of Physiology and Gastrointestinal Liver Physiology, Vol. 303, No. 9, 2012, pp. G1029-G1104. doi:10.1152/ajpgi.00003.2012

[9] C. L. Ohland and W. K. Macnaughton, "Probiotic Bacteria and Intestinal Epithelial Barrier Function,” American Journal of Physiology and Gastrointestinal Liver Physiology, Vol. 298, No. 6, 2010, pp G807-G819. doi:10.1152/ajpgi.00243.2009

[10] F. J. Penna, L. A. Peret, L. Q. Vieira and J. R. Nicoli, "Probiotics and Mucosal Barrier in Children,” Current Opinion in Clinical Nutrition and Metabolic Care, Vol. 11, No. 5, 2008, pp. 640-644. doi:10.1097/MCO.0b013e32830a70ab

[11] R. Mennigen and M. Bruewer, "Effect of Probiotics on Intestinal Barrier Function,” Annals of the New York Aca- demy of Science, Vol. 1165, 2009, pp. 183-189. doi:10.1111/j.1749-6632.2009.04059.x

[12] K. Teahon, S. Somasundaram, T. Smith, I. Menzies and I. Bjarnason, "Assessing the Site of Increased Intestinal Permeability in Coeliac and Inflammatory Bowel Disease," Gut, Vol. 38, No. 6, 1996, pp. 864-869. doi:10.1136/gut.38.6.864

[13] L. Miele, et al., "Increased Intestinal Permeability and Tight Junction Alterations in Nonalcoholic Fatty Liver Disease," Hepatology, Vol. 49, No. 6, 2009, pp. 1877 1887. doi:10.1002/hep. 22848

[14] A. Parlesak, C. Schafer, T. Schutz, J. C. Bode and C. Bode, "Increased Intestinal Permeability to Macromolecules and Endotoxemia in Patients with Chronic Alcohol Abuse in Different Stages of Alcohol-Induced Liver Disease,” Journal of Hepatology, Vol. 32, No. 5, 2000, pp. 742-747. doi:10.1016/S0168-8278(00)80242-1

[15] B. J. Ammori, et al., "Early Increase in Intestinal Permeability in Patients with Severe Acute Pancreatitis: Correlation with Endotoxemia, Organ Failure, and Mortality," Journal of Gastrointestinal Surgery, Vol. 3, No. 3, 1999, pp. 252-261.doi:10.1016/S1091-255X(99)80067-5

[16] H. Lennernas, "Human Intestinal Permeability,” Journal of Pharmaceutical Sciences, Vol. 87, No. 4, 1998, pp. 403-410. doi:10.1021/js970332a

[17] M. G. Besselink, et al., "Intestinal Barrier Dysfunction in a Randomized Trial of a Specific Probiotic Composition in Acute Pancreatitis,” Annals of Surgery, Vol. 250, No. 5, 2009, pp. 712-719. doi:10.1097/SLA.0b013e3181bce5bd

[18] G. M. Diepenhorst, et al., "Influence of Prophylactic Probiotics and Selective Decontamination on Bacterial Translocation in Patients Undergoing Pancreatic Surgery: A Randomized Controlled Trial,” Shock, Vol. 35, No. 1, 2011, pp. 9-16. doi:10.1097/SHK.0b013e3181ed8f17

[19] T. Kanda, et al., "Intestinal Fatty Acid-Binding Protein Is a Useful Diagnostic Marker for Mesenteric Infarction in Humans," Gastroenterology, Vol. 110, No. 2, 1996, pp. 339-343. doi:10.1053/gast.1996.v110.pm8566578

[20] J. M. Lieberman, J. Sacchettini, C. Marks and W. H. Marks, "Human Intestinal Fatty Acid Binding Protein: Report of an Assay with Studies in Normal Volunteers and Intestinal Ischemia,” Surgery, Vol. 121, No. 3, 1997, pp. 335-342. doi:10.1016/S0039-6060(97)90363-9

[21] A. Fasano, et al., "Zonulin, a Newly Discovered Modulator of Intestinal Permeability, and Its Expression in Coeliac Disease,” Lancet, Vol. 355, No. 9214, 2000, pp. 1518-1519. doi:10.1016/S0140-6736(00)02169-3

[22] W. L. Wang, S. Uzzau, S. E. Goldblum and A. Fasano, "Human Zonulin, a Potential Modulator of Intestinal Tight Junctions,” Journal of Cell Science, Vol. 113, No. 24, 2000, pp. 4435-4440.

[23] A. Tripathi, et al., "Identification of Human Zonulin, a Physiological Modulator of Tight Junctions, as Prehaptoglobin-2," Proceedings of the National Academy of Sciences of the United States of America, Vol. 106, No. 39, 2009, pp. 16799-16804. doi:10.1073/pnas.0906773106

[24] F. Lutgendorff, “Defending the Barrier," Ph.D. Thesis, Universiteit Utrecht, Utrecht, 2009.

[25] M. Lamprecht, et al., "Probiotic Supplementation Affects 
Markers of Intestinal Barrier, Oxidation, and Inflammation in Trained Men; A Randomized, Double-Blinded, Placebo-Controlled Trial,” Journal of the International Society of Sports Nutrition, Vol. 9, No. 1, 2012, p. 45. doi:10.1186/1550-2783-9-45

[26] M. Viljanen, et al., "Probiotic Effects on Faecal Inflammatory Markers and on Faecal Iga in Food Allergic Atopic Eczema/Dermatitis Syndrome Infants,” Pediatric Allergy and Immunology, Vol. 16, No. 1, 2005, pp. 65-71. doi:10.1111/j.1399-3038.2005.00224.X

[27] C. Alberda, et al., "Effects of Probiotic Therapy in Critically Ill Patients: A Randomized, Double-Blind, PlaceboControlled Trial," American Journal of Clinical Nutrition, Vol. 85, No. 3, 2007, pp. 816-823.

[28] E. Garcia Vilela, et al., "Influence of Saccharomyces Boulardii on the Intestinal Permeability of Patients with Crohn's Disease in Remission,” Scandanavian Journal of Gastroenterology, Vol. 43, No. 7, 2008, pp. 842-848. doi:10.1080/00365520801943354

[29] M. Gotteland, S. Cruchet and S. Verbeke, "Effect of Lactobacillus Ingestion on the Gastrointestinal Mucosal Barrier Alterations Induced by Indometacin in Humans," Alimentary and Pharmacology Therapy, Vol. 15, No. 1, 2001, pp. 11-17. doi:10.1046/j.1365-2036.2001.00898.x

[30] Z. Liu, et al., "Randomised Clinical Trial: The Effects of Perioperative Probiotic Treatment on Barrier Function and Post-Operative Infectious Complications in Colorectal Cancer Surgery-A Double-Blind Study," Alimentary Pharmacology \& Therapeutics, Vol. 33, No. 1, 2011, pp. 50-63. doi:10.1111/j.1365-2036.2010.04492.x

[31] C. E. McNaught, N. P. Woodcock, J. MacFie and C. J. Mitchell, "A Prospective Randomised Study of the Probiotic Lactobacillus Plantarum 299v on Indices of Gut Barrier Function in Elective Surgical Patients,” Gut, Vol. 51, No. 6, 2002, pp. 827-831. doi:10.1136/gut.51.6.827

[32] B. S. Reddy, et al., "Randomized Clinical Trial of Effect of Synbiotics, Neomycin and Mechanical Bowel Preparation on Intestinal Barrier Function in Patients Undergoing Colectomy,” British Journal of Surgery, Vol. 94, No. 5, 2007, pp. 546-554. doi:10.1002/bjs.5705

[33] Z. Stratiki, et al., "The Effect of a Bifidobacter Supplemented Bovine Milk on Intestinal Permeability of Preterm Infants,” Early Human Development, Vol. 83, No. 9, 2007, pp. 575-579.

[34] J. W. Zhang, et al., "Preoperative Probiotics Decrease Postoperative Infectious Complications of Colorectal Cancer," American Journal of Medical Science, Vol. 343, No. 3, 2012, pp. 199-205. doi:10.1097/MAJ.0b013e31823aace6

[35] J. Karczewski, et al., "Regulation of Human Epithelial Tight Junction Proteins by Lactobacillus Plantarum in Vivo and Protective Effects on the Epithelial Barrier," American Journal of Physiology and Gastrointestinal Liver Physiology, Vol. 298, No. 6, 2010, pp G851-G859. doi:10.1152/ajpgi.00327.2009

[36] R. J. Boyle, F. J. Bath-Hextall, J. Leonardi-Bee, D. F. Murrell and M. L. Tang, "Probiotics for the Treatment of Eczema: A Systematic Review," Clinical and Experimental Allergy, Vol. 39, No. 8, 2009, pp. 1117-1127. doi:10.1111/j.1365-2222.2009.03305.X

[37] D. Jonkers, J. Penders, A. Masclee and M. Pierik, "Probi- otics in the Management of Inflammatory Bowel Disease: A Systematic Review of Intervention Studies in Adult Patients,” Drugs, Vol. 72, No. 6, 2012, pp. 803-823. doi:10.2165/11632710-000000000-00000

[38] P. Moayyedi, et al., "The Efficacy of Probiotics in the Treatment of Irritable Bowel Syndrome: A Systematic Review,” Gut, Vol. 59, No. 3, 2010, pp. 325-332. doi:10.1136/gut.2008.167270

[39] T. A. Sentongo, et al., "Intestinal Permeability and Effects of Lactobacillus rhamnosus Therapy in Children with Short Bowel Syndrome," Journal of Pediatric Gastroenterology and Nutrition, Vol. 46, No. 1, 2008, pp. 4147. doi:10.1097/01.mpg.0000304452.92175.f5

[40] P. Gupta, H. Andrew, B. S. Kirschner and S. Guandalini, "Is Lactobacillus GG Helpful in Children with Crohn's Disease? Results of a Preliminary, Open-Label Study," Journal of Pediatric Gastroenterology and Nutrition, Vol. 31, No. 4, 2000, pp. 453-457. doi:10.1097/00005176-200010000-00024

[41] E. Isolauri, M. Juntunen, T. Rautanen, P. Sillanaukee and T. Koivula, “A Human Lactobacillus Strain (Lactobacillus casei SP Strain GG) Promotes Recovery from Acute Diarrhea in Children,” Pediatrics, Vol. 88, No. 1, 1991, pp. 90-97.

[42] B. Leber, et al., "The Influence of Probiotic Supplementation on Gut Permeability in Patients with Metabolic Syndrome: An Open Label, Randomized Pilot Study," European Journal of Clinical Nutrition, Vol. 66, No. 10, 2012, pp. 1110-1115. doi:10.1038/ejcn.2012.103

[43] B. Sharma, et al., "Role of Probiotics on Gut Permeability and Endotoxemia in Patients with Acute Pancreatitis: A Double-Blind Randomized Controlled Trial,” Journal of Clinical Gastroenterology, Vol. 45, No. 5, 2011, pp. 442448. doi:10.1097/MCG.0b013e318201f9e2

[44] C. Gore, et al., "Treatment and Secondary Prevention Effects of the Probiotics Lactobacillus paracasei or Bifidobacterium lactis on Early Infant Eczema: Randomized Controlled Trial with Follow-Up until Age 3 Years,” Clinical and Experimental Allergy, Vol. 42, No. 1, 2012, pp. 112-122. doi:10.1111/j.1365-2222.2011.03885.x

[45] H. L. Qin, et al., "Effect of Lactobacillus plantarum Enteral Feeding on the Gut Permeability and Septic Complications in the Patients with Acute Pancreatitis," European Journal of Clinical Nutrition, Vol. 62, No. 7, 2008, pp. 923-930. doi:10.1038/sj.ejcn.1602792

[46] R. Francavilla, et al., "A Randomized Controlled Trial of Lactobacillus GG in Children with Functional Abdominal Pain,” Pediatrics, Vol. 126, No. 6, 2010, pp. e1445-e1452. doi:10.1542/peds.2010-0467

[47] R. C. Anderson, A. L. Cookson, W. C. McNabb, W. J. Kelly and N. C. Roy, "Lactobacillus Plantarum DSM 2648 Is a Potential Probiotic That Enhances Intestinal Barrier Function,” FEMS Microbiology Letters, Vol. 309, No. 2, 2010, pp. 184-192. doi:10.1111/j.1574-6968.2010.02038.x

[48] C. M. Chapman, G. R. Gibson and I. Rowland, "In Vitro Evaluation of Single- and Multi-Strain Probiotics: InterSpecies Inhibition between Probiotic Strains, and Inhibition of Pathogens," Anaerobe, Vol. 18, No. 4, 2012, pp. 405-413. doi:10.1016/j.anaerobe.2012.05.004

[49] H. M. Timmerman, C. J. Koning, L. Mulder, F. M. Rom- 
bouts and A. C. Beynen, "Monostrain, Multistrain and Multispecies Probiotics-A Comparison of Functionality and Efficacy," International Journal of Food Microbiology, Vol. 96, No. 3, 2004, pp. 219-233.

doi:10.1016/j.ijfoodmicro.2004.05.012

[50] M. Nermes, J. M. Kantele, T. J. Atosuo, S. Salminen and E. Isolauri, "Interaction of Orally Administered Lactobacillus rhamnosus GG with Skin and Gut Microbiota and Humoral Immunity in Infants with Atopic Dermatitis," Clinical and Experimental Allergy, Vol. 41, No. 3, 2011, pp. 370-377. doi:10.1111/j.1365-2222.2010.03657.x

[51] C. J. O’Boyle, et al., “Alterations in Intestinal Barrier Function Do Not Predispose to Translocation of Enteric Bacteria in Gastroenterologic Patients,” Nutrition, Vol. 14, No. 4, 1998, pp. 358-362. doi:10.1016/S0899-9007(97)00488-7

[52] M. Lamprecht and A. Frauwallner, "Exercise, Intestinal Barrier Dysfunction and Probiotic Supplementation," $\mathrm{Me}$ dical Sport Science, Vol. 59, 2013, pp. 47-56. doi:10.1159/000342169

[53] E. J. Schiffrin, et al., "Probiotic Yogurt in the Elderly with Intestinal Bacterial Overgrowth: Endotoxaemia and Innate Immune Functions,” British Journal of Nutrition, Vol. 101, No. 7, 2009, pp. 961-966. doi:10.1017/S0007114508055591

[54] M. A. van Nieuwenhoven, B. J. Geerling, N. E. Deutz, F. Brouns and R. J. Brummer, "The Sensitivity of the Lactulose/Rhamnose Gut Permeability Test,” European Journal of Clinical Investigations, Vol. 29, No. 2, 1999, pp. 160-165. doi:10.1046/j.1365-2362.1999.00421.x

[55] F. Scaldaferri, M. Pizzoferrato, V. Gerardi, L. Lopetuso and A. Gasbarrini, "The Gut Barrier: New Acquisitions and Therapeutic Approaches,” Journal of Clinical Gastroenterology, Vol. 46, 2012, pp. S12-S17. doi:10.1097/MCG.0b013e31826ae849

[56] D. R. Duerksen, C. Wilhelm-Boyles, R. Veitch, D. Kryszak and D. M. Parry, "A Comparison of Antibody Testing, Permeability Testing, and Zonulin Levels with SmallBowel Biopsy in Celiac Disease Patients on a GlutenFree Diet," Digestive Disease Science, Vol. 55, No. 4, 2010, pp. 1026-1031. doi:10.1007/s10620-009-0813-5

[57] A. Sapone, et al., "Zonulin Upregulation Is Associated with Increased Gut Permeability in Subjects with Type 1 Diabetes and Their Relatives,” Diabetes, Vol. 55, No. 5, 2006, pp. 1443-1449. doi:10.2337/db05-1593

[58] K. Becker, T. Frieling and D. Haussinger, "Quantification of Fecal Alpha 1-Antitrypsin Excretion for Assessment of Inflammatory Bowel Diseases," European Journal of Medical Research, Vol. 3, No. 1-2, 1998, pp. 65-70.

[59] S. Y. Salim and J. D. Soderholm, "Importance of Disrupted Intestinal Barrier in Inflammatory Bowel Diseases,” Inflammatory Bowel Disease, Vol. 17, No. 1, 2011, pp. 362-381. doi:10.1002/ibd.21403

[60] L. Biancone, et al., "Non-Invasive Techniques for Assessing Postoperative Recurrence in Crohn's Disease," Digestive Liver Diseases, Vol. 40, Suppl. 2, 2008, pp. S265-S270. doi:10.1016/S1590-8658(08)60536-8

[61] K. Tiihonen, A. C. Ouwehand and N. Rautonen, "Effect of Overweight on Gastrointestinal Microbiology and Immunology: Correlation with Blood Biomarkers,” British
Journal of Nutrition, Vol. 103, No. 7, 2009, pp. 1-9. doi:10.1017/S0007114509992807

[62] J. Holland, et al., "Intraoperative Splanchnic Hypoperfusion, Increased Intestinal Permeability, Down-Regulation of Monocyte Class II Major Histocompatibility Complex Expression, Exaggerated Acute Phase Response, and Sepsis,” American Journal of Surgery, Vol. 190, No. 3, 2005, pp. 393-400. doi:10.1016/j.amjsurg.2005.03.038

[63] Z. F. Tang, Y. B. Ling, N. Lin, Z. Hao and R. Y. Xu, "Glutamine and Recombinant Human Growth Hormone Protect Intestinal Barrier Function Following Portal Hypertension Surgery," World Journal of Gastroenterology, Vol. 13, No. 15, 2007, pp. 2223-2228.

[64] J. Brignardello, et al., "Pilot Study: Alterations of Intestinal Microbiota in Obese Humans Are Not Associated with Colonic Inflammation or Disturbances of Barrier Function,” Alimentary Pharmacology \& Therapeutics, Vol. 32, No. 11-12, 2010, pp. 1307-1314. doi:10.1111/j.1365-2036.2010.04475.x

[65] V. Rosenfeldt, et al., "Effect of Probiotics on Gastrointestinal Symptoms and Small Intestinal Permeability in Children with Atopic Dermatitis," Journal of Pediatrics, Vol. 145, No. 5, 2004, pp. 612-616. doi:10.1016/j.jpeds.2004.06.068

[66] B. Klarin, et al., "Lactobacillus Plantarum 299v Reduces Colonisation of Clostridium Difficile in Critically Ill Patients Treated with Antibiotics,” Acta Anaesthesiologica Scandinavica, Vol. 52, No. 8, 2008, pp. 1096-1102. doi:10.1111/j.1399-6576.2008.01748.x

[67] J. Zeng, et al., "Clinical Trial: Effect of Active Lactic Acid Bacteria on Mucosal Barrier Function in Patients with Diarrhoea-Predominant Irritable Bowel Syndrome,” Alimentary Pharmacology \& Therapeutics, Vol. 25, No. 8, 2008 pp. 994-1002. doi:10.1111/j.1365-2036.2008.03818.x

[68] L. A. Gonzalez-Hernandez, et al., "Synbiotic Therapy Decreases Microbial Translocation and Inflammation and Improves Immunological Status in HIV-Infected Patients: a Double-Blind Randomized Controlled Pilot Trial,” $\mathrm{Nu}$ trition Journal, Vol. 11, 2012, p. 90. doi:10.1186/1475-2891-11-90

[69] P. K. Jain, C. E. Mcnaught, A. D. Anderson, J. Macfie and C. J. Mitchell, "Influence of Synbiotic Containing Lactobacillus acidophilus La5, Bifidobacterium lactis Bb 12, Streptococcus thermophilus, Lactobacillus bulgaricus and Oligofructose on Gut Barrier Function and Sepsis in Critically Ill Patients: A Randomised Controlled Trial,” Clinical Nutrition, Vol. 23, No. 4, 2004, pp. 467-475. doi:10.1016/j.clnu.2003.12.002

[70] C. E. Mcnaught, N. P. Woodcock, A. D. Anderson and J. Macfie, "A Prospective Randomised Trial of Probiotics in Critically Ill Patients,” Clinical Nutrition, Vol. 24, No. 2, 2005, pp. 211-219. doi:10.1016/j.clnu.2004.08.008

[71] L. Galpin, et al., "Effect of Lactobacillus GG on Intestinal Integrity in Malawian Children at Risk of Tropical Enteropathy," American Journal of Clinical Nutrition, Vol. 82, No. 5, 2005, pp. 1040-1045.

[72] M. Schunter, et al., "Randomized Pilot Trial of a Synbiotic Dietary Supplement in Chronic HIV-1 Infection," BMC Complementary and Alternative Medicine, Vol. 12, 2012, p. 84. doi:10.1186/1472-6882-12-84 